\title{
Post marketing surveillance on medical implants in neurosurgery
}

\author{
M.J. Staal* \\ Academic Hospital, Department of Neurosurgery, Hanzeplein 1, P.O. Box 30001, 9700 RB \\ Groningen, The Netherlands
}

Neurosurgeons deal with the most vulnerable human organ, the central and peripheral nervous system (CNS and PNS): the brain and its efferent and afferent neural structures (spinal cord), and peripheral nerves. Also, the CNS blood vessel system as well as the CNS surrounding structures, like the membranes covering the CNS tissue and the bony elements enveloping the CNS (skull and spinal column), belong to the neurosurgeon's field of work. The very precarious character of the neurosurgeon's thinking and handling is mainly determined by a poor reversibility of lost neurological functions due to his intervention. However, this is not always an unwanted consequence of his handling but sometimes done intentionally.

Neurosurgical activities focus on both congenital and acquired anomalies, like:

- tumors of the nervous system and its surrounding tissues;

- vascular anomalies within the nervous system;

- degenerative (e.g., slipped disc), traumatic (e.g., fractures) and developmental disorders of the skull and spinal column;

- intracranial haemorrhages;

- disturbances of the circulation of the cerebro-spinal fluid (CSF);

- compression of nerves;

- developmental midline defects, e.g., spina bifida; and

- infectious disorders.

Most of these can be made visible directly by modern radiological imaging techniques, like computer tomography (CT scan) and magnetic resonance imaging (MRI).

Besides intervention in these "visible targets", some neurosurgeons are working in the field of socalled functional neurosurgery. In this part of the profession the neurosurgeon intentionally interferes in a "function" of the nervous system, or in other terms modulates a "function". In these cases, however, this "function" has a pathological nature, and always extends beyond physiological limits. These pathological functions or malfunctions, mostly being an element of an underlying disease, are undesired, seriously disturbing a patient's quality of life.

\footnotetext{
${ }^{*}$ Neurosurgeon.
} 
Examples of these malfunctions are:

- pain (which by origin is not a pathological function!);

- movement disorders, like tremor in Parkinson's disease or multiple sclerosis (MS);

- spasticity (which, in contrast to its unphysiological nature, sometimes can be very functional!), as seen after spinal column fractures or in MS; and

- epilepsy.

In both fields of neurosurgery three types of implants are used:

- biological implants (which are subject of rapidly growing interest in neurosurgery);

- dural substitution materials (synthetical and biological); and

- mechanical implants.

The latter are presented in this paper and can be distinguished in:

- vascular clips,

- ventricular shunt systems,

- spinal cord and brain stimulating systems,

- spinal column osteosynthesis devices, and

- drug administration systems.

In neurosurgical practice, registration of these implants is not systematically done by neurosurgeons, neither in The Netherlands nor abroad. The Dutch Association of Neurosurgeons has been invited by the SIG Health Care Information, Utrecht, The Netherlands, to participate in a project for registration of medical implants, which is already in development for orthopaedic and plastic surgery. The author has been asked to function as intermediate. His personal experience fully underlines the necessity of neurosurgical participation in such a registration system, because of the following reasons:

- the usefulness of some of the currently used neurosurgical implants has not been fully proven yet;

- differences in quality and/or safety of comparable systems have not yet been thoroughly examined; and

- in an individual patient implant retrieval can gain important information about previous neurosurgical interventions performed elsewhere.

In general terms a neurosurgical implant retrieval system can therefore serve:

1. Clinical research on the indications of the application of several implant systems, especially CNS stimulation and drug administration systems;

2. Research on quality and reliability of different implants, especially CSF regulating devices; and

3. Individual and collective patient directed care in case of missing information (individually) or in case of recall of unsafe or unreliable implants (collectively).

However, benefits depend on several factors such as the rate of cooperation and participation of the potential users (with an estimated minimum of 90\%), the amount of work to be done in the participating centres and close agreement between the users and the management of the data system about access possibilities and patient privacy. 